
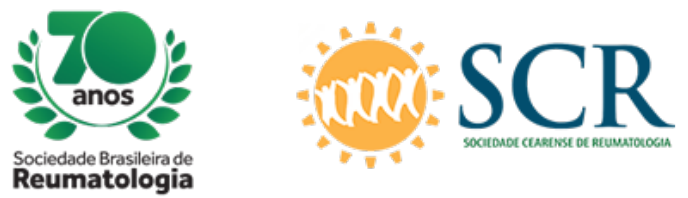

\title{
TOXIC EPIDERMAL NECROLYSIS TRIGGERED BY GLUCOCORTICOIDS AND PAROTITIS AS FIRST MANIFESTATION OF SYSTEMIC LUPUS ERYTHEMATOSUS- CASE REPORT
}

Débora Rocha de Moura Rodrigues de Aguiar (Hospital Federal de Bonsucesso, Rio de Janeiro, RJ, Brasil), Luiz Felipe Dipe (Hospital Federal de Bonsucesso, Rio de Janeiro, RJ, Brasil), Anna Maria Amaral de Oliveira (Hospital Federal de Bonsucesso, Rio de Janeiro, RJ, Brasil), Thamires Castro da Silva Cardoso

(Hospital Federal de Bonsucesso, Rio de Janeiro, RJ, Brasil), Elba Sophia Theodoro Santos de Oliveira (Hospital Federal de Bonsucesso, Rio de Janeiro, RJ, Brasil), Luciana de Abreu e Lima Pamplona (Hospital Federal de Bonsucesso, Rio de Janeiro, RJ, Brasil)

\section{BACKGROUND}

Systemic Lupus Erythematosus (SLE) is a heterogeneous disease, with systemic presentation, that can affect joints, skin, kidneys, heart, lungs, nervous system, and immune system. Toxic epidermal necrolysis (TEN) can be a life threatening mucocutaneous reaction, usually associated with drug exposure antibiotics, antiepileptics, allopurinol and NSAIDs, as well as infections, more rare causes: SLE and vaccines. It is characterized by extensive necrosis and skin and mucous sloughing involving more than $30 \%$ of the body surface. Although TEN is a rare presentation of Systemic Lupus Erythematosus (SLE), it does form the SLICC 2012 classification criteria. Parotiditis is an infrequent manifestation of SLE. It is described as a secondary presentation of Sjogren's Syndrome or in juvenile SLE.

\section{CASE REPORT}

A 49-year-old black man, without pre-existing diseases and did not use drugs regularly. Present adynamia, bilateral parotid enlargement and crusted skin lesions on the face, back and thorax. It has evolved with diffuse face and parotid edema, associated with face rash back and thorax. Denied xerophthalmia, xerostomia, arthralgia or photosensitivity. Echocardiogram with mild pericardial effusion. Computerized tomography shows lymph nodes enlargement between 2 and 3,5 cm at neck, thorax and abdomen and pleural effusion. Ultrasonography of parotid gland shows heterogeneity. Laboratory tests showed coarse nuclear speckled ANA 1: 640, anti-DNA, anti-Ro and anti-La were negative; anti-Sm 29.68 (positive $>25$ ) and anti-RNP $>200$ (positive $>25$ ). After 2 days of treatment with glucocorticoids at a dose of $1 \mathrm{mg}$ per kilogram of body weight, the patient developed blistering of the face, back and thorax (fig.1) associated with oral ulcers and fever, without genital involvement. Histopatologic of skin revealed TEN. He presented hypotension, hemodynamic instability, requiring intensive care. We have prescribed intravenous immune globulin (IVIG). He presented regression of the cutaneous involvement and parotids reduction. There was involution of the parotid enlargement not being possible the accomplishment of histopathological.

\section{CONCLUSION}

Parotitis and TEN-like are rare manifestations in SLE. In our case, no association with Sjogren's Syndrome has been demonstrated. Another differential of our case is the presentation TEN-like after beginning of the systemic corticoterapia. TEN can be associated with hydroxychloroquine and glucocorticoides, in rare cases. We highlight the response a IVIG, an option for treatment as well as cyclosporine. It is described in the literature the increase in survival with IVIG.

\section{Consent for publication}

The authors declare that the patient was oriented and signed the written informed consent form. 